\title{
The Regularized Mesh Scheme to Solve Quasilinear Parabolic Equation with Time-Fractional Derivative
}

\author{
A. V. Lapin ${ }^{1 *}$ and E. Laitinen ${ }^{2 * *}$ \\ (Submitted by A. M. Elizarov) \\ ${ }^{1}$ Department of Higher Mathematics, Mechanics, and Mathematical Modelling, \\ Institute of Personalized Medicine, Sechenov University, Moscow, 119435 Russia \\ ${ }^{2}$ Faculty of Science, Research Unit of Mathematical Sciences, University of Oulu, Oulu, Finland
}

Received March 1, 2021; revised March 20, 2021; accepted March 25, 2021

\begin{abstract}
A quasilinear parabolic problem with a time fractional derivative of the Caputo type and mixed boundary conditions is considered. The coefficients of the elliptic operator depend on the gradient of the solution, and this operator is uniformly monotone and Lipschitz-continuous. For this problem, unconditionally stable linear regularized semi-discrete scheme is constructed based on the $L 1$-approximation of the fractional time derivative. Stability estimates are obtained by the variational method. Accuracy estimates are given provided that the initial data and the solution to the differential problem are sufficiently smooth. The proved result of stability of the semi-discrete scheme is valid for the mesh scheme obtained from the semi-discrete problem using the finite element method in spatial variables.
\end{abstract}

DOI: $10.1134 / \mathrm{S} 1995080221070155$

Keywords and phrases: quasilinear parabolic problem, time-fractional derivative, regularized scheme, stability, accuracy.

\section{INTRODUCTION}

Partial differential equations with time-fractional derivatives arise in mathematical modeling of anomalous diffusion and dynamic processes in materials with memory. Numerous articles are devoted to the approximation of boundary value problems for linear problems with time-fractional derivatives. In recent years, the numerical methods has been actively developed for equations with time-fractional derivatives and the elliptic parts containing the non-linearities. Thus, implicit mesh schemes for the equations with Caputo time-fractional derivatives and with right-hand sides depending on the solution are studied in [1-3]. In [4] an implicit scheme is investigated for the nonlinear diffusion equation with a coefficient depending on the solution and with Caputo time-fractional derivative. In [5-10], analysis of ADI schemes was performed for linear time-fractional equations and for a class of semilinear timefractional equations.

The article [11] proposes a new mathematical model for a viscoelastic-plastic process by formulating a temporary fractional equation containing an elliptic operator, which depends on the gradient of the solution. In [12], the authors construct and study backward Euler and locally one dimensional schemes approximating Dirichlet problem with Caputo time-fractional derivative and a quasilinear elliptic part without mixed derivatives.

In this article, we consider a quasilinear parabolic problem with a time fractional derivative of the Caputo type and mixed boundary conditions. The quasilinear elliptic part of the problem defines a uniformly monotone and Lipschitz operator, which is energy equivalent to the Laplace operator. For this problem, we construct and investigate a linear regularized semidiscrete scheme in which the regularization term contains the Laplace operator and time-fractional derivative is approximated using

\footnotetext{
*E-mail: avlapine@mail.ru

***-mail: erkki.laitinen@oulu.fi
} 
well-known L1-approximation. Stability estimates for the semidiscrete problem by the variational method are obtained. Accuracy estimates are given provided that the initial data and the solution to the differential problem are sufficiently smooth. For discrete schemes obtained as a result of mesh approximations of the elliptic part of the semidiscrete problem, the stability estimates remain valid. They are also generalized for factorized schemes.

\section{FORMULATION OF THE PROBLEM}

Let $\Omega \subset \mathbb{R}^{2}$ be a polygon with the boundary $\partial \Omega=\Gamma_{D} \cup \Gamma_{N}$, meas $\Gamma_{D}>0$, and $Q=\Omega \times(0, T]$, $\Sigma_{D}=\Gamma_{D} \times(0, T], \Sigma_{N}=\Gamma_{N} \times(0, T]$. Define a Caputo-type time-fractional derivative:

$$
\mathcal{D}_{t} y(t)=\int_{0}^{t} G(t-s) \frac{\partial y}{\partial s}(s) d s
$$

with a kernel $G(t)$ satisfying the assumptions:

$$
G(t):(0,+\infty) \rightarrow \mathbb{R}^{+} \text {is continuous and strictly decreasing function, } \quad \int_{0}^{+\infty} G(t) d t<\infty .
$$

The conditions (2) are satisfied for

- the generalized Caputo fractional derivative with $G(t)=\frac{r(t)}{\Gamma(1-\alpha) t^{\alpha}}, 0<\alpha<1, \Gamma(x)$ is gammafunction, a weighting function $r(t) \in C^{2}[0, T], r(t)>0$ and $r^{\prime}(t) \leqslant 0$ for all $t \in[0, T](r(t) \equiv 1$ corresponds to the classical Caputo fractional derivative);

- the multi-term fractional derivative with $G(t)=\sum_{k=1}^{s} \frac{c_{k}}{\Gamma\left(1-\alpha_{k}\right) t^{\alpha_{k}}}, \quad 0<\alpha_{1}<\ldots<\alpha_{s}<1$, $c_{k}>0$.

See the definitions of the corresponding derivatives, e.g., in [13-15]).

Let us define a second order quasilinear elliptic differential operator $L$ and the co-normal derivative $\frac{\partial u}{\partial \nu_{L}}$ by the equalities

$$
L u=-\sum_{i=1}^{2} \frac{\partial}{\partial x_{i}} g_{i}(x, t, u, \nabla u)+g_{0}(x, t, u, \nabla u), \quad \frac{\partial u}{\partial \nu_{L}}=\sum_{i=1}^{2} g_{i}(x, t, u, \nabla u) \cos \left(n, x_{i}\right),
$$

where $n$ is the unit vector of the outward normal to $\Sigma_{N}$. Suppose that the nonlinear coefficients $g_{i}(t, p)=g_{i}\left(x, t, p_{0}, p_{1}, p_{2}\right)$ of the equation satisfy the following assumptions for all $(x, t) \in \bar{Q}$ and $p, q, r \in \mathbb{R}^{3}$ :

$$
\begin{gathered}
g_{i}(t, p), \quad \text { for } i, k=0, \ldots, 2 \text { are continuous, } \\
\sum_{i=0}^{2}\left(g_{i}(t, p)-g_{i}(t, q)\right)\left(p_{i}-q_{i}\right) \geqslant c_{0} \sum_{i=1}^{2}\left(p_{i}-q_{i}\right)^{2}, \quad c_{0}>0, \\
\sum_{i=0}^{2}\left(g_{i}(t, p)-g_{i}(t, q)\right) r_{i} \leqslant c_{1}\left(\sum_{i=0}^{2}\left(g_{i}(t, p)-g_{i}(t, q)\right)\left(p_{i}-q_{i}\right)\right)^{1 / 2}\left(\sum_{i=0}^{2} r_{i}^{2}\right)^{1 / 2} .
\end{gathered}
$$

Consider a quasilinear parabolic problem with the time-fractional derivative and mixed boundary conditions:

$$
\mathcal{D}_{t} u+L u=f \quad \text { in } \quad Q_{T},
$$




$$
\begin{gathered}
u=0 \text { on } \Sigma_{D}, \quad \frac{\partial u}{\partial \nu_{L}}=q \text { on } \Sigma_{N}, \\
u=0 \text { for } t=0, \quad x \in \Omega .
\end{gathered}
$$

We assume that given functions $f=f(x, t)$ and $q=q(x, t)$ are continuous in $t$ in order to use the simplest mesh approximations and do not specify their properties in details, since we do not investigate the existence of a solution to problem (5).

Let us make only a few remarks about well-known results on the existence of weak solutions of parabolic equations with time-fractional derivatives. The existence of a regular weak solution to Dirichlet problem for the case of linear operator $L$ and classical Caputo fractional derivative was proved in [16]. A priori estimate for the solution in the norm of $H^{\alpha}\left(0, T ; L^{2}(\Omega)\right) \cap L^{2}\left(0, T ; H_{0}^{1}(\Omega) \cap H^{2}(\Omega)\right)$ through the $L^{2}(Q)$-norm of $f$ was derived. The existence of a weak solution from Besov space $B^{\alpha / 2}\left(Q_{T}\right)=H^{\alpha / 2}\left(0, T ; L^{2}(\Omega)\right) \cap L^{2}\left(0, T ; H^{1}(\Omega)\right)$ is substantiated in [12] for a quasilinear Dirichlet boundary value problem with classical Caputo derivative and a particular case of differential operator (3). This result can be generalized for the case of boundary value problem with elliptic operator (3) and Caputo time derivative. In a general case the problem of the existence of a unique solution to (5) seems to be open. In the future, we will assume that problem (5) has a smooth solution, and will focus on studying the mesh approximation of this problem.

\section{APPROXIMATION}

Multiplying the differential equation (5) by a sufficiently smooth function $v(x):\left.v\right|_{\Gamma_{D}}=0$, we obtain a variational equation that can serve as a basis for the constructing its approximations:

$$
\int_{\Omega} \mathcal{D}_{t} u v d x+\int_{\Omega}\left(\sum_{i=1}^{2} g_{i}(x, t, u, \nabla u) \frac{\partial v}{\partial x_{i}}+g_{0}(x, t, u, \nabla u) v\right) d x=\int_{\Omega} f v d x+\int_{\Gamma_{N}} q v d \Gamma .
$$

Let $V=\left\{y \in H^{1}(\Omega) ; y=0\right.$ a.e. $\left.\quad x \in \Gamma_{D}\right\}$ with the norm $\|u\|_{V}^{2}=\int_{\Omega}\left(u^{2}+|\nabla u|^{2}\right) d x$, and let $\|u\|_{0}^{2}=\int_{\Omega} u^{2} d x,\|u\|_{1}^{2}=\int_{\Omega}|\nabla u|^{2} d x$ and $\|u\|_{\Gamma}^{2}=\int_{\Gamma_{N}} u^{2} d \Gamma$. It is well-known that

$$
\|u\|_{0} \leqslant c_{\Omega}\|u\|_{1}, \quad\|v\|_{\Gamma} \leqslant c_{\Gamma}\|v\|_{1} \quad \forall v \in V .
$$

Due to the first inequality in (6) the norms $\|u\|_{V}$ and $\|u\|_{1}$ are equivalent. By virtue of this fact and the assumptions (4) the form

$$
a(t, u, v)=\int_{Q_{T}}\left(\sum_{i=1}^{2} g_{i}(x, t, u, \nabla u) \frac{\partial v}{\partial x_{i}}+g_{0}(x, t, u, \nabla u) v\right) d x d t
$$

is well-defined on $V \times V$ for all $t$ and has the following properties:

$$
\begin{array}{cl}
a(t, u, v-u)-a(t, v, v-u) \geqslant c_{0}\|u-v\|_{1}^{2} & \forall u, v \in V, \\
a(t, u-v, w) \leqslant c_{2}\|u-v\|_{1}\|w\|_{1} \quad \forall u, v, w \in V, & c_{2}=c_{2}\left(c_{1}, c_{\Omega}\right) .
\end{array}
$$

Let $\omega_{\tau}=\left\{t_{j}=j \tau, j=0,1, \ldots M ; M \tau=T\right\}$ be a uniform mesh on the segment $[0, T]$ and let us use the notation $y^{j}=y\left(t_{j}\right)$ for a continuous function $y(t)$. The $L 1$-approximation of the time derivative $\mathcal{D}_{t} y(t)=\int_{0}^{t} G(t-s) \frac{\partial y}{\partial s}(s) d s$ of a continuous function $y(t)$ at a point $t_{k} \in \omega_{\tau}$ is given by the following relations:

$$
\mathcal{D}_{t} y\left(t_{k}\right) \approx \partial_{t} \bar{y}^{k}=d_{1} y^{k}+\sum_{j=1}^{k-1}\left(d_{j+1}-d_{j}\right) y^{k-j}-d_{k} y^{0}
$$




$$
d_{j}=\frac{1}{\tau} \int_{t_{k-j}}^{t_{k-j+1}} G\left(t_{k}-s\right) d s=\frac{1}{\tau} \int_{(j-1) \tau}^{j \tau} G(u) d u, \quad \bar{y}^{k}=\left(y^{0}, y^{1}, \ldots, y^{k}\right) .
$$

The properties (2) imply that the coefficients satisfy the inequalities

$$
d_{1}>d_{2}>\cdots>d_{M}>0 .
$$

First, we consider the semidiscrete implicit scheme approximating problem (5): find $\left(y\left(x, t_{1}\right), y\left(x, t_{2}\right), \ldots, y\left(x, t_{M}\right)\right)$ with $y\left(x, t_{k}\right) \in V$ and $y(x, 0)=0$, such that for all $k$

$$
\int_{\Omega} \partial_{t} \bar{y}^{k}(x) v(x) d x+a\left(t_{k}, y^{k}, v\right)=\int_{\Omega} f\left(x, t_{k}\right) v(x) d x+\int_{\Gamma_{N}} q\left(x, t_{k}\right) v d \Gamma \quad \forall v \in V .
$$

Since $\partial_{t} \bar{y}^{k}(x)=d_{1} y^{k}(x)+\sum_{j=1}^{k-1}\left(d_{j+1}-d_{j}\right) y^{k-j}(x)$, then a $k$-th equation of system $(9)$ is a variational equation

$$
\int_{\Omega} d_{1} y^{k}(x) v(x) d x+a\left(t_{k}, y^{k}, v\right)=F_{k}(v)
$$

with right-hand side

$$
F_{k}(v)=\int_{\Omega} f\left(x, t_{k}\right) v(x) d x+\int_{\Gamma_{N}} q\left(x, t_{k}\right) v d \Gamma-\sum_{j=1}^{k-1}\left(d_{j+1}-d_{j}\right) \int_{\Omega} \nabla y^{k-j}(x) \cdot \nabla v(x) d x .
$$

Due to the properties (7) the left side of (10) defines a continuous and uniformly monotone operator from $V$ to its dual $V^{*}$. Using the inequalities (6), it is easy to see that linear functional $F_{k} \in V^{*}$ for $f\left(x, t_{k}\right) \in L^{2}(\Omega)$ and $q\left(x, t_{k}\right) \in L^{2}\left(\Gamma_{N}\right)$. Thus, the existence of a unique solution to problem (10) follows from [17], Chapter 2, section 2.1.

Below, instead of the nonlinear problem (9), a linear regularized problem is constructed. To shorten writing, we will use the notations

$$
(u, v)=\int_{\Omega} u v d x, \quad a_{k}(u, v)=a\left(t_{k}, u, v\right) \quad\left(f^{k}, v\right)=\int_{\Omega} f\left(x, t_{k}\right) v(x) d x+\int_{\Gamma_{N}} q\left(x, t_{k}\right) v d \Gamma .
$$

Let us introduce a bilinear bounded form $r(u, v)$ on the space $V \times V$, which satisfies the inequality

$$
r(u, u) \geqslant\|u\|_{1}^{2} \quad \forall u \in V .
$$

A particular case of this bilinear form is $r(u, v)=\int_{\Omega} \nabla u \cdot \nabla v d x$ for $u, v \in V$.

The regularized scheme approximating (5) is as follows: for a given $y^{0}=0$ find $y^{k}$ for $k \geqslant 1$ from the system of linear equations

$$
\left(\partial_{t} \bar{y}^{k}, v\right)+\sigma r\left(y^{k}-y^{k-1}, v\right)+a\left(t_{k-1}, y^{k-1}, v\right)=\left(f^{k}, v\right) \quad \forall v \in V, \quad \sigma>0 .
$$

For a fixed $k$ the mesh scheme (12) can be written as

$$
d_{1}\left(y^{k}, v\right)+\sigma r\left(y^{k}-y^{k-1}, v\right)+\sum_{j=1}^{k-1}\left(d_{j+1}-d_{j}\right)\left(y^{k-j}, v\right)+a_{k-1}\left(y^{k-1}, v\right)=\left(f^{k}, v\right),
$$

The linear variational problem (13) has a unique solution for any $\sigma \geqslant 0$ by Lax-Milgram theorem.

The essential point used in proving stability estimates is the property of the $L 1$-approximation of the time-fractional derivative, which is given by the following lemma: 
Lemma 1 ([12]). Let

$$
D=\left(\begin{array}{cccccccc}
d_{2} & 0 & 0 & 0 & \cdots & 0 & 0 & 0 \\
0 & d_{2} & 0 & 0 & \cdots & 0 & 0 & 0 \\
d_{3}-d_{2} & 0 & d_{2} & 0 & \cdots & 0 & 0 & 0 \\
\cdots & \cdots & \cdots & \cdots & \cdots & \cdots & \cdots & \\
d_{M}-d_{M-1} & d_{M-1}-d_{M-2} & d_{M-2}-d_{M-3} & \cdots & \cdots & d_{3}-d_{2} & 0 & d_{2}
\end{array}\right) .
$$

Then the symmetric matrix $1 / 2\left(D+D^{T}\right)$ is positive definite.

Using the matrix $D$, the approximation of time derivative at a point $t_{k}=k \tau \in \omega_{\tau}$ can be written as

$$
\partial_{t} y\left(t_{k}\right)=\left(d_{1}-d_{2}\right)\left(y^{k}-y^{k-1}\right)+\left(D_{k} \bar{y}^{k}\right)^{k}
$$

where $D_{k}$ is $k \times k$ leading submatrix of $B$, and $1 / 2\left(D_{k}+D_{k}^{T}\right)$ is positive definite along with $1 / 2\left(D+D^{T}\right)$ :

$$
\sum_{k=1}^{m}\left(D_{k} \bar{y}^{k}\right)^{k} y^{k} \geqslant 0 \quad \forall \bar{y}^{k}=\left(y^{1}, y^{2}, \ldots, y^{k}\right) .
$$

Introduce also the bilinear, symmetric and positive definite form on $V \times V$ :

$$
b(u, v)=\left(d_{1}-d_{2}\right)(u, v)+\sigma r(u, v) .
$$

Now the equation (13) can be rewritten as

$$
b\left(y^{k}-y^{k-1}, v\right)+\left(\left(D_{k} \bar{y}^{k}\right)^{k}, v\right)+a_{k-1}\left(y^{k-1}, v\right)=\left(f^{k}, v\right) .
$$

For generality, we derive an a priori estimate for the case of a nonzero initial value. For $i=1,2$ we denote by $y_{i}$ the solutions of the equation (13) with the right-hand sides $f_{i}$ and the initial values $y_{i}^{0}$. Let also $y=y_{1}-y_{2}, y^{0}=y_{1}^{0}-y_{2}^{0}$ and $\psi=f_{1}-f_{2}$.

Lemma 2. Let the conditions (7) be satisfied and the regularization parameter satisfies the condition

$$
\sigma=\frac{c_{2}^{2}}{2 c_{0}}+\delta, \delta>0
$$

Then there is a constant $\mu=\mu(\delta)$ such that for all $m=1,2, \ldots$ M the following a priori estimate for the regularized mesh scheme (13) holds:

$$
\left(d_{1}-d_{2}\right)\left\|y^{m}\right\|_{0}^{2}+\mu \sum_{k=1}^{m}\left\|y^{k}\right\|_{1}^{2} \leqslant\left\|y^{0}\right\|_{0}^{2}+r\left(y^{0}, y^{0}\right)+2 \sum_{k=1}^{m}\left|\left(\psi^{k}, y^{k}\right)\right| .
$$

Proof. From the equations (24) written for $y_{1}^{k}$ and $y_{2}^{k}$ with test function $v=2\left(y_{1}^{k}-y_{2}^{k}\right)=2 y^{k}$ we obtain:

$$
2 b\left(y^{k}-y^{k-1}, y^{k}\right)+2\left(\left(D_{k} \bar{y}^{k}\right)^{k}, y^{k}\right)+2 a_{k-1}\left(y_{1}^{k-1}, v\right)-2 a_{k-1}\left(y_{1}^{k-1}, y^{k}\right)=2\left(\psi^{k}, y^{k}\right) .
$$

In what follows, we use the following estimates:

$$
\begin{gathered}
2 b\left(y^{k}-y^{k-1}, y^{k}\right)=\left\|y^{k}\right\|_{b}^{2}-\left\|y^{k-1}\right\|_{b}^{2}+\left\|y^{k}-y^{k-1}\right\|_{b}^{2} \\
2 a_{k-1}\left(y_{1}^{k-1}, y^{k}\right)-2 a_{k-1}\left(y_{2}^{k-1}, y^{k}\right) \\
=2 a_{k-1}\left(y_{1}^{k-1}, y^{k-1}+y^{k}-y^{k-1}\right)-2 a_{k-1}\left(y_{2}^{k-1}, y^{k-1}+y^{k}-y^{k-1}\right) \\
\geqslant 2 c_{0}\left\|y^{k-1}\right\|_{1}^{2}-2 c_{2}\left\|y^{k-1}\right\|_{1}\left\|y^{k}-y^{k-1}\right\|_{1} \geqslant\left(2 c_{0}-\delta_{0}\right)\left\|y^{k-1}\right\|_{1}^{2}-\frac{c_{2}^{2}}{\delta_{0}}\left\|y^{k}-y^{k-1}\right\|_{1}^{2}
\end{gathered}
$$

with a constant $\delta_{0} \in\left(0,2 c_{0}\right)$ in the second estimate. 
Using the derived estimates and the inequality (14), after summing the equation (18) over $k$ from 1 to $m$ we obtain

$$
\begin{aligned}
\left\|y^{m}\right\|_{b}^{2} & -\left\|y^{0}\right\|_{b}^{2}+\sum_{k=1}^{m}\left(\left\|y^{k}-y^{k-1}\right\|_{b}^{2}-\frac{c_{2}^{2}}{\delta_{0}}\left\|y^{k}-y^{k-1}\right\|_{1}^{2}\right) \\
& +\left(2 c_{0}-\delta_{0}\right) \sum_{k=1}^{m}\left\|y^{k-1}\right\|_{1}^{2} \leqslant 2 \sum_{k=1}^{m}\left|\left(\psi^{k}, y^{k}\right)\right| .
\end{aligned}
$$

Now, due to the definition of the bilinear form $b(.,$.$) and the inequalities (11) and (16) we have the$ inequality

$$
\left\|y^{k}-y^{k-1}\right\|_{b}^{2} \geqslant \sigma\left\|y^{k}-y^{k-1}\right\|_{1}^{2}=\left(\frac{c_{2}^{2}}{2 c_{0}}+\delta\right)\left\|y^{k}-y^{k-1}\right\|_{1}^{2} .
$$

With an appropriate choice of the constant $\delta_{0}=\delta_{0}(\delta) \in\left(0,2 c_{0}\right)$, we obtain

$$
\left\|y^{k}-y^{k-1}\right\|_{b}^{2}-\frac{c_{2}^{2}}{\delta_{0}}\left\|y^{k}-y^{k-1}\right\|_{1}^{2} \geqslant\left(\frac{c_{2}^{2}}{2 c_{0}}+\delta-\frac{c_{2}^{2}}{\delta_{0}(\delta)}\right)\left\|y^{k}-y^{k-1}\right\|_{1}^{2} \geqslant 0,
$$

whence

$$
\left\|y^{m}\right\|_{b}^{2}-\left\|y^{0}\right\|_{b}^{2}+\left(2 c_{0}-\delta_{0}(\delta)\right) \sum_{k=1}^{m}\left\|y^{k-1}\right\|_{1}^{2} \leqslant 2 \sum_{k=1}^{m}\left|\left(\psi^{k}, y^{k}\right)\right| .
$$

Let $\mu(\delta)=\min \left\{\sigma, 2 c_{0}-\delta_{0}(\delta)\right\}$. Since $\left\|y^{m}\right\|_{b}^{2}=\left(d_{1}-d_{2}\right)\left\|y^{m}\right\|^{2}+\sigma\left\|y^{m}\right\|_{1}^{2}$, then the last inequality implies (17)

The a priori estimate (17) can be used to obtain various stability estimates for the regularized mesh scheme (13). We give one such estimate in the case of zero initial condition. Let

$$
\|f\|_{-1}=\sup _{u \in V \backslash\{0\}} \frac{(f, u)}{\|u\|_{1}}=\sup _{u \in V \backslash\{0\}} \frac{\int_{\Omega} f u d x}{\left(\int_{\Omega}|\nabla u|^{2} d x\right)^{1 / 2}}
$$

and let the following norms be defined for the functions from $\omega_{\tau}$ to $V$ or to its subspace $W$ :

$$
\|y\|_{L^{2}\left(\omega_{\tau} ; H^{1}\right)}^{2}=\sum_{k=1}^{M} \tau\left\|y^{k}\right\|_{1}^{2}, \quad\|f\|_{L^{2}\left(\omega_{\tau} ; H^{-1}\right)}^{2}=\sum_{k=1}^{M} \tau\left\|u^{k}\right\|_{-1}^{2}, \quad\|q\|_{L^{2}\left(\omega_{\tau} ; L^{2}(\Gamma)\right)}^{2}=\sum_{k=1}^{M} \tau\left\|u^{k}\right\|_{\Gamma}^{2} .
$$

Theorem 1. Let the conditions (7) be satisfied, $y_{i}$ be the solutions of the equation (13) with the right-hand sides $f_{i}$, homogeneous initial conditions $y_{i}^{0}=0$ and the right-hand sides in Neuman boundary conditions $q_{i}$. If the regularization parameter satisfies the condition (16):

$$
\sigma=\frac{c_{2}^{2}}{2 c_{0}}+\delta, \delta>0
$$

then the following stability estimate holds:

$$
\left\|y_{1}-y_{2}\right\|_{L^{2}\left(\omega_{\tau} ; H^{1}\right)} \leqslant C\left(\left\|f_{1}-f_{2}\right\|_{L^{2}\left(\omega_{\tau} ; H^{-1}\right)}+\left\|q_{1}-q_{2}\right\|_{L^{2}\left(\omega_{\tau} ; L^{2}(\Gamma)\right)}\right), \quad C=C(\delta) .
$$

Proof. The inequality (17) implies

$$
\mu \sum_{k=1}^{m} \tau\left\|y_{1}^{k}-y_{2}^{k}\right\|_{1}^{2} \leqslant 2 \sum_{k=1}^{m} \tau\left|\left(\psi^{k}, y_{1}^{k}-y_{2}^{k}\right)\right|
$$

with

$$
\left(\psi^{k}, v\right)=\int_{\Omega}\left(f_{1}\left(x, t_{k}\right)-f_{2}\left(x, t_{k}\right)\right) v(x) d x+\int_{\Gamma_{N}}\left(q_{1}\left(x, t_{k}\right)-q_{2}\left(x, t_{k}\right) v(x) d \Gamma .\right.
$$


For all $k$ and $v \in V$

$$
\left|\int_{\Omega}\left(f_{1}\left(x, t_{k}\right)-f_{2}\left(x, t_{k}\right)\right) v(x) d x\right| \leqslant\left\|f_{1}^{k}-f_{2}^{k}\right\|_{-1}\|v\|_{1}
$$

and due to (6)

$$
\mid \int_{\Gamma_{N}}\left(q_{1}\left(x, t_{k}\right)-q_{2}\left(x, t_{k}\right) v(x) d \Gamma \mid \leqslant\left\|q_{1}^{k}-q_{2}^{k}\right\|_{\Gamma}\|v\|_{\Gamma} \leqslant c_{\Gamma}\left\|q_{1}^{k}-q_{2}^{k}\right\|_{\Gamma}\|v\|_{1} .\right.
$$

Now the estimate (20) immediately follows from (21) and the last two inequalities.

Using the proven stability estimate, we can obtain an accuracy estimate under the assumption that the input data and the solution to the differential problem are sufficiently smooth. Let $y^{k}, k=$ $1,2, \ldots, M$, be the solution of a semidiscrete problem $(12)$ and $u^{k}=u\left(x, t_{k}\right)$ be the exact solution $u(x, t)$ of the differential problem (5). Then the estimate (18) applied to the difference $y-u$ contains in the right-hand side the function $\psi^{k}$ which is defined by the equality

$$
\left(\psi^{k}, v\right)=\left(\partial_{t} \bar{u}^{k}-\mathcal{D}_{t} u\left(t_{k}\right), v\right)+\sigma r\left(u^{k}-u^{k-1}, v\right) .
$$

Let us consider the case of classical Caputo derivative. The following estimate is well-known ([19, 20]): $\partial_{t}^{\alpha} \bar{u}^{k}=\mathcal{D}_{t}^{\alpha} u\left(t_{k}\right)+O\left(\tau^{2-\alpha}\right)$. It is easy to see that $r\left(u^{k}-u^{k-1}, v\right)=\tau r\left(\frac{u^{k}-u^{k-1}}{\tau}, v\right) \leqslant O(\tau)\|v\|_{1}$. Thus, the most significant term in the approximation error is associated with the introduction of the regularizing term, and the accuracy estimate is

$$
\|y-u\|_{L^{2}\left(\omega_{\tau} ; H^{1}\right)}=O(\tau) .
$$

Note that for the implicit scheme (backward Euler scheme) and locally one-dimensional scheme (see [12]), the terms in the accuracy estimate related to the approximation in time have the following orders respectively:

$$
\|y-u\|_{L^{2}\left(\omega_{\tau} ; H^{1}\right)}=O\left(\tau^{2-\alpha}\right), \quad\|y-u\|_{L^{2}\left(\omega_{\tau} ; L^{2}\right)}=O\left(\tau^{\alpha / 2}\right) .
$$

Both of these schemes are nonlinear and require the use of iterative methods when implemented at each time level.

Remark 1. A fully discrete approximation of problem (5) can be constructed using an approximation with respect to the spatial variables of the semidiscrete problem (12). All the results on the existence of a unique solution and the proved stability estimates remain valid for mesh problems constructed using the finite element method with finite elements of the first order, as well as for finite element schemes with quadrature formulas.

Remark 2. The explicit (forward Euler) mesh scheme is a particular case of fully discrete regularized scheme with $\sigma=0$. A sufficient condition of its stability follows from the estimate (19):

$$
\left(d_{1}-d_{2}\right)\|u\|^{2} \geqslant \frac{c_{2}^{2}}{\delta_{0}}\|u\|_{1}^{2} \quad \forall u \in V .
$$

The last inequality is equivalent to the following estimate for the maximum eigenvalue $\lambda_{\max }$ of the mesh Laplace operator:

$$
d_{1}-d_{2} \geqslant \lambda_{\max }
$$

As is known, $\lambda_{\max }=O\left(h^{-2}\right)$, where $h$ characterizes the mesh step in the spatial variable. Since, for example, for the $L 1$-approximation of the Caputo derivative, $d_{1}-d_{2}=O\left(\tau^{-\alpha}\right)$, then (22) leads to a very restrictive condition for the smallness of the time step:

$$
\tau<c h^{2 / \alpha}
$$

with a constant $c$, which depends on the problem data. 
Remark 3. Let $r_{1}(u, v)$ be a bounded bilinear form in $V \times V$ and $r_{1}(u, u) \geqslant 0$. Then the following scheme satisfies all the proven results on unique solvability and stability:

$$
\left(\partial_{t} \bar{y}^{k}, v\right)+\sigma r\left(y^{k}-y^{k-1}, v\right)+r_{1}\left(y^{k}, v\right)+a\left(t_{k-1}, y^{k-1}, v\right)=\left(f^{k}, v\right) \quad \forall v \in V, \quad \sigma>0 .
$$

This fact allows to apply these results to the factorized mesh schemes. More precisely, let for a fully discrete scheme constructed using finite element or finite difference scheme $r(u, v)=(R u, v)$ and the mesh Laplace operator $R=-\Delta_{h}$ with corresponding boundary conditions can be splitted into the sum $R=R_{1}+R_{2}$ such that $R_{1} R_{2}$ is a non-negative mesh operator: $\left(R_{1} R_{2} u, u\right) \geqslant 0$. In this case we set $r_{1}(u, v)=\left(R_{1} R_{2} u, v\right)$ and take the regularization term in the form

$$
\sigma r\left(y^{k}-y^{k-1}, v\right)+\sigma^{2} d_{1}^{-1} r_{1}\left(y^{k}, v\right) .
$$

The corresponding mesh scheme becomes the mesh scheme with a factorized operator on each time level:

$$
\begin{gathered}
d_{1}\left(\left(I+\sigma d_{1}^{-1} R_{1}\right)\left(I+\sigma d_{1}^{-1} R_{2}\right) y^{k}, v\right)-\sigma\left(R y^{k-1}, v\right)+\sum_{j=1}^{k-1}\left(d_{j+1}-d_{j}\right)\left(y^{k-j}, v\right) \\
+a_{k-1}\left(y^{k-1}, v\right)=\left(f^{k}, v\right), \quad I \text { is the identity operator. }
\end{gathered}
$$

The implementation of (24) is reduced to sequential inversion of the mesh operators $I+\sigma d_{1}^{-1} R_{1}$ and $I+\sigma d_{1}^{-1} R_{2}$ and is very easy if these operators are locally one-dimensional (that corresponds to alternating direction method) or triangle (that corresponds to alternating triangular method). The term $\sigma^{2} d_{1}^{-1} r_{1}\left(u^{k}, v\right)$ is added to the approximation error. In case of classical Caputo derivative it is asymptotically $O\left(\tau^{\alpha}\right)$. So, the accuracy estimate is

$$
\|y-u\|_{L^{2}\left(\omega_{\tau} ; H^{1}\right)}=O\left(\tau^{\alpha}\right) .
$$

\section{FUNDING}

This research was supported by Russian Foundation of Basic Researches, project no. 19-01-00431 and Academy of Finland, grant no. 333448 (Alexander Lapin) and Academy of Finland, grant no. 333551 (Erkki Laitinen).

\section{OPEN ACCESS}

This article is licensed under a Creative Commons Attribution 4.0 International License, which permits use, sharing, adaptation, distribution and reproduction in any medium or format, as long as you give appropriate credit to the original author(s) and the source, provide a link to the Creative Commons license, and indicate if changes were made. The images or other third party material in this article are included in the article's Creative Commons license, unless indicated otherwise in a credit line to the material. If material is not included in the article's Creative Commons license and your intended use is not permitted by statutory regulation or exceeds the permitted use, you will need to obtain permission directly from the copyright holder. To view a copy of this license, visit http://creativecommons.org/licenses/by/4.0/.

\section{REFERENCES}

1. B. Jin, B. Li, and Z. Zhou, "Numerical analysis of nonlinear subdiffusion equations," SIAM J. Numer. Anal. 56, 1-23(2018).

2. D. Li, H.-L. Liao, W. Sun, J. Wang, and J. Zhang, "Analysis of L1-Galerkin FEMs for time-fractional nonlinear parabolic problems," Commun. Comput. Phys. 24, 86-103 (2018).

3. D. Li, J. Zhang, and Z. Zhang, "Unconditionally optimal orror Estimates of a linearized Galerkin method for nonlinear time fractional reaction-subdiffusion equations," J. Sci. Comput. 76, 848-866 (2018).

4. D. N. Gerasimov, V. A. Kondratieva, and O. A. Sinkevich, "An anomalous non-self-similar infiltration and fractional diffusion equation," Phys. D (Amsterdam, Neth.) 239, 1593-1597 (2010).

5. Y. Zhang, Z. Sun, and X. Zhao, "Compact alternating direction implicit scheme for the two dimensional fractional diffusion-wave equation,” SIAM J. Numer. Anal. 50, 1535-1555 (2002). 
6. A. Chen and C. Li, "A novel compact ADI scheme for the time-fractional subdiffusion equation in two space dimensions," Int. J. Comput. Math. 93, 889-914 (2016).

7. G. H. Gao and Z. Z. Sun, "Two alternating direction implicit difference schemes for two-dimensional distributed-order fractional diffusion equations," J. Sci. Comput. 66, 1281-1312 (2016).

8. P. Zhu and S. L. Xie, "ADI finite element method for 2D nonlinear time fractional reaction-subdiffusion equation,” Am. J. Comput. Math. 6, 336-356 (2016).

9. W. Qiu, Da Xu, H. Chen, and J. Guo, "An alternating direction implicit Galerkin finite element method for the distributed-order time-fractional mobile-immobile equation in two dimensions," Comput. Math. Appl. 80, 3156-3172 (2020).

10. H. Jiang, D. Xu, W. Qiu, and J. Zhou, "An ADI compact difference scheme for the two-dimensional semilinear time-fractional mobile-immobile equation," Comput. Appl. Math. 39 (287) (2020).

11. S. Tatar, R. Tnaztepe, and M. Zeki, "Numerical solutions of direct and inverse problems for a time fractional viscoelastoplastic equation,” J. Eng. Mech. 143 (7) (2017).

12. A. Lapin and K. Levinskaya, "Numerical solution of a quasilinear parabolic equation with a fractional time derivative," Lobachevskii J. Math. 41 (12), 2673-2686 (2020).

13. T. Sandev, A. Chechkin, H. Kantz, and R. Metzler, "Diffusion and Fokker-Planck-Smoluchowski equations with generalized memory kernel," Fract. Calc. Appl. Anal. 18, 1006-1038 (2015).

14. B. Jin, R. Lazarov, Y. Liu, and Z. Zhou, "The Galerkin finite element method for a multi-term time-fractional diffusion equation," J. Comput. Phys. 281, 825-843 (2015).

15. Gh. Gao, A. A. Alikhanov, and Z. Sun, "The temporal second order difference schemes based on the interpolation approximation for solving the time multi-term and distributed-order fractional sub-diffusion equations,” J. Sci. Comput. 73, 93-121 (2017).

16. R. Gorenflo, Yu. Luchko, and M. Yamamoto, "Time-fractional diffusion equation in the fractional Sobolev spaces," Fract. Calc. Appl. Anal. 18, 799-820 (2015).

17. J.-L. Lions, Quelques méthodes de résolution des problèmes aux limites non linéaires (Paris, Dunod, 1969).

18. Ph. G. Ciarlet, The Finite Element Method for Elliptic Problems (North-Holland, Amsterdam, 1978).

19. T. A. M. Langlands and B. I. Henry, "The accuracy and stability of an implicit solution method for the fractional diffusion equation," J. Comput. Phys. 205, 719-736 (2005).

20. Y. Lin and C. Xu, "Finite difference/spectral approximations for the time-fractional diffusion equation," J. Comput. Phys. 225, 1552-1553 (2007). 